\title{
A METHOD FOR VISUAL IDENTIFICATION OF SMALL SAMPLE SUBGROUPS AND POTENTIAL BIOMARKERS - SUPPLEMENTARY MATERIAL
}

\author{
By Charlotte Soneson and Magnus Fontes \\ Lund University
}

1. A small example. Figure S1 shows the steps of CUMBIA when applied to a small synthetic example consisting of four samples (S1-S4) and five variables (V1-V5). For details, see the figure caption.

2. Emphasizing both over- and underexpressed variables. In its basic formulation, CUMBIA emphasizes variables which are jointly highly overexpressed in a group of samples. It can be adjusted to detect also unusually low expression values for some variables in a group of samples. For example, assuming the variables are centered, formula (6) in the main paper can be replaced by

$$
\begin{gathered}
d_{s}\left(\mathbf{s}_{i}, \mathbf{s}_{j}\right)=\min \left(\min _{1 \leq k \leq p}\left(\sqrt{\lambda_{1}-\left(X_{s}^{T}\right)_{k i}}+\sqrt{\lambda_{1}-\left(X_{s}^{T}\right)_{k j}}\right),\right. \\
\left.\min _{1 \leq k \leq p}\left(\sqrt{\lambda_{1}-\left(-X_{s}^{T}\right)_{k i}}+\sqrt{\lambda_{1}-\left(-X_{s}^{T}\right)_{k j}}\right)\right), \\
1 \leq i, j \leq N, \mathbf{s}_{i} \neq \mathbf{s}_{j} \\
(1) d_{s}\left(\mathbf{w}_{i}, \mathbf{w}_{j}\right)=\min \left(\min _{1 \leq k \leq p}\left(\sqrt{\lambda_{1}-\left(X_{s}\right)_{k i}}+\sqrt{\lambda_{1}-\left(X_{s}\right)_{k j}}\right),\right. \\
\left.\min _{1 \leq k \leq p}\left(\sqrt{\lambda_{1}-\left(-X_{s}\right)_{k i}}+\sqrt{\lambda_{1}-\left(-X_{s}\right)_{k j}}\right)\right), \\
1 \leq i, j \leq p, \mathbf{w}_{i} \neq \mathbf{w}_{j} \\
d_{s}\left(\mathbf{s}_{i}, \mathbf{w}_{j}\right)=\sqrt{\lambda_{1}-\left(X_{s}\right)_{i j}}, \\
1 \leq i \leq N, 1 \leq j \leq p .
\end{gathered}
$$

This implies that also variables sharing an unusually low value (e.g. a negative $z$-score) in a sample will be considered similar to each other, while still being considered dissimilar to the sample. Intuitively, it corresponds to flipping the sign of the centered data matrix, calculating all sample-sample and variable-variable dissimilarities again, and for each pair of samples (or variables) keeping the smallest of the two calculated dissimilarities (corresponding to the two sign choices of the data matrix). This dissimilarity can 
C. SONESON AND M. FONTES
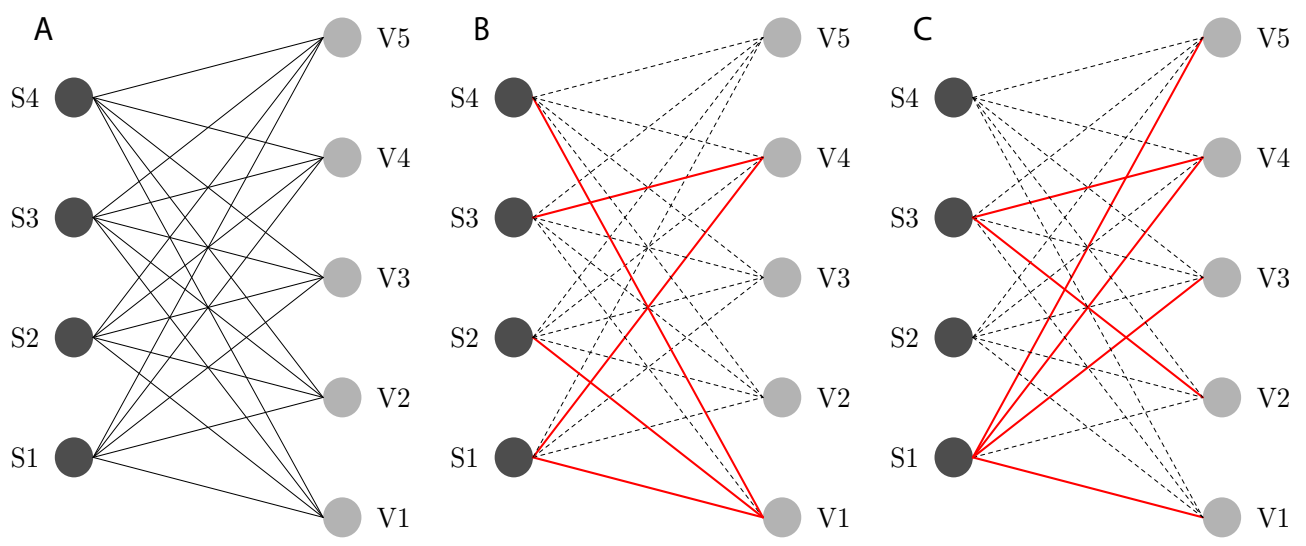

$$
X=\left(\begin{array}{ccccc}
8 & -2 & 3 & 4 & 3 \\
9 & 1 & 2 & -2 & 3 \\
-2 & 2 & 1 & 4 & 2 \\
-1 & -2 & 3 & 2 & -1
\end{array}\right) \quad \tilde{D}=\left(\begin{array}{ccccc}
2.311 & 3.916 & 3.215 & 3.056 & 3.215 \\
2.083 & 3.513 & 3.367 & 3.916 & 3.215 \\
3.916 & 3.367 & 3.513 & 3.056 & 3.367 \\
3.787 & 3.916 & 3.215 & 3.367 & 3.787
\end{array}\right)
$$

$\lambda_{1}=13.339$

$$
D=\left(\begin{array}{ccccccccc}
0 & 4.394 & 6.112 & 6.097 & 2.311 & 3.916 & 3.215 & 3.056 & 3.215 \\
4.394 & 0 & 6.000 & 5.870 & 2.083 & 3.513 & 3.367 & 3.916 & 3.215 \\
6.112 & 6.000 & 0 & 6.423 & 3.916 & 3.367 & 3.513 & 3.056 & 3.367 \\
6.097 & 5.870 & 6.423 & 0 & 3.787 & 3.916 & 3.215 & 3.367 & 3.787 \\
2.311 & 2.083 & 3.916 & 3.787 & 0 & 5.596 & 5.450 & 5.367 & 5.298 \\
3.916 & 3.513 & 3.367 & 3.916 & 5.596 & 0 & 6.880 & 6.423 & 6.728 \\
3.215 & 3.367 & 3.513 & 3.215 & 5.450 & 6.880 & 0 & 6.271 & 6.431 \\
3.056 & 3.916 & 3.056 & 3.367 & 5.367 & 6.423 & 6.271 & 0 & 6.271 \\
3.215 & 3.215 & 3.367 & 3.787 & 5.298 & 6.728 & 6.431 & 6.271 & 0
\end{array}\right)
$$

FIG S1. A small example showing the steps of CUMBIA. Panel A shows the bipartite graph resulting from connecting each variable to each sample. The expression matrix is denoted by $X$ and the largest singular value is $\lambda_{1}=13.339$. $\tilde{D}$ denotes the matrix of dissimilarities between samples and variables (i.e. $\tilde{D}_{i j}=\sqrt{\lambda_{1}-X_{i j}}$ ) that is used to compute samplesample and variable-variable dissimilarities. Panel $B$ shows the shortest paths between sample $S 1$ and the other samples marked in red (we choose $K=1$ and consider only overexpressed variables), and panel $C$ shows the shortest paths between variable $V 4$ and each of the other variables marked in red. Finally, the CUMBIA dissimilarity matrix is given by $D$. 
also be extended to the mean value of $K$ paths to increase the robustness. These $K$ paths may represent either only overexpressed variables, only underexpressed variables or a combination of the two types. If the researcher wishes to emphasize only underexpressed variables, the sign of the entire centered data matrix may be changed. Then, jointly underexpressed variables will be placed close to the corresponding samples.

We evaluate this adjustment on a data set created from the cell culture data described in the main article by including, for the cardiac stromal cells and umbilical artery endothelial cells, 25 variables which are highly positively discriminative and 25 variables which are highly negatively discriminative for each group compared to the rest of the samples. We also include 1,500 variables having the least discriminative power contrasting all tissue/cell type groups. Figure $\mathrm{S} 2$ shows the low-dimensional representations of the samples and variables obtained by CUMBIA (panel A) and PCA (panel B). In panel A, we note that CUMBIA is able to represent both the over- and underexpressed variable groups for the two sample groups so that they can be visually extracted. Interestingly, the second CUMBIA component now characterizes a single sample, and a group of variables which are jointly underexpressed in this sample only.

3. Using different values of $\boldsymbol{K}$. The choice of the number of paths to average over $(K)$ when computing the CUMBIA dissimilarities can have a major impact on the resulting visualization. Using a small value of $K$ we are able to detect small groups of samples and variables which are closely related, but the algorithm becomes sensitive to noisy measurements. Increasing $K$ makes the algorithm more robust, but in this case larger groups of samples and variables are favored. Figure S3 shows the resulting visualizations when we apply CUMBIA to the cell culture data studied in the main article, using $K \in\{3,10,30\}$. We note that as $K$ is increased, it becomes more difficult to detect the small sample and variable groups.

4. Using approximate expression values to calculate CUMBIA dissimilarities. In the main paper, we calculated the CUMBIA dissimilarity matrices based on the ( $\log _{2}$-transformed) measured expression values. We also discussed the possibility of using approximate values, given by the optimal rank- $s$ approximation to $X$ obtained by SVD, as a basis for the dissimilarity calculations. This may be advantageous from a noise reduction point of view. However, it is important to note that choosing a too small value of $s$, we may discard interesting features of the data. In Figure S4 we show the effect of choosing $s=5$ and $s=15$ (the rank of the original matrix is 60) compared to using $X$ for the cell culture data studied in the 

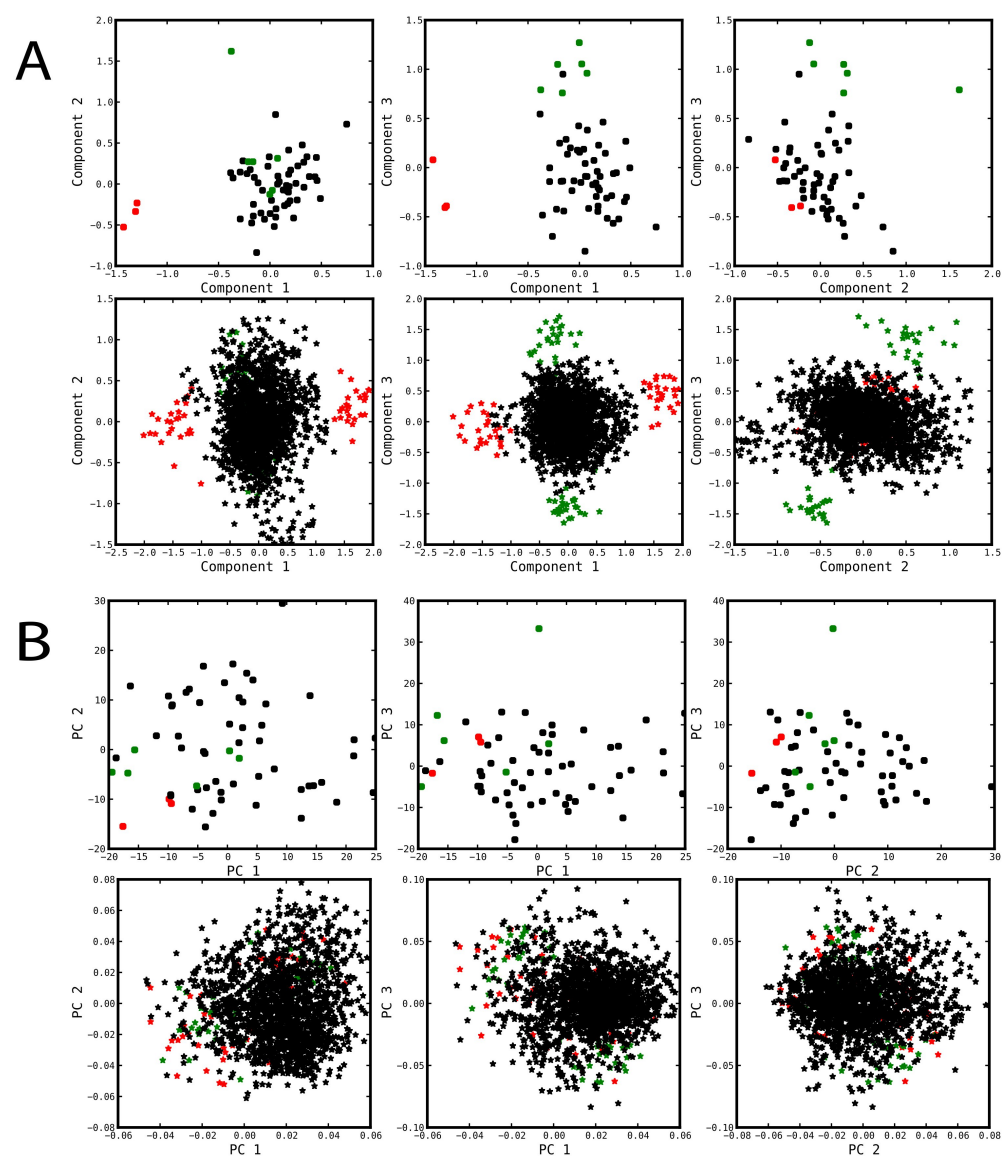

FIG S2. Low-dimensional representation of samples and variables from the human cell cultures microarray data set, obtained by CUMBIA (panel A) and PCA (panel B). Green markers represent samples from umbilical artery endothelial cells, and the 50 variables chosen to have high (positive and negative) discriminative power for these samples compared to the rest. Similarly, red markers represent cardiac stromal cell samples and variables. Black markers represent samples from all other subgroups, and the 1,500 variables from the original data set which are least discriminating when contrasting all tissue/cell type combinations in the data set. 
A
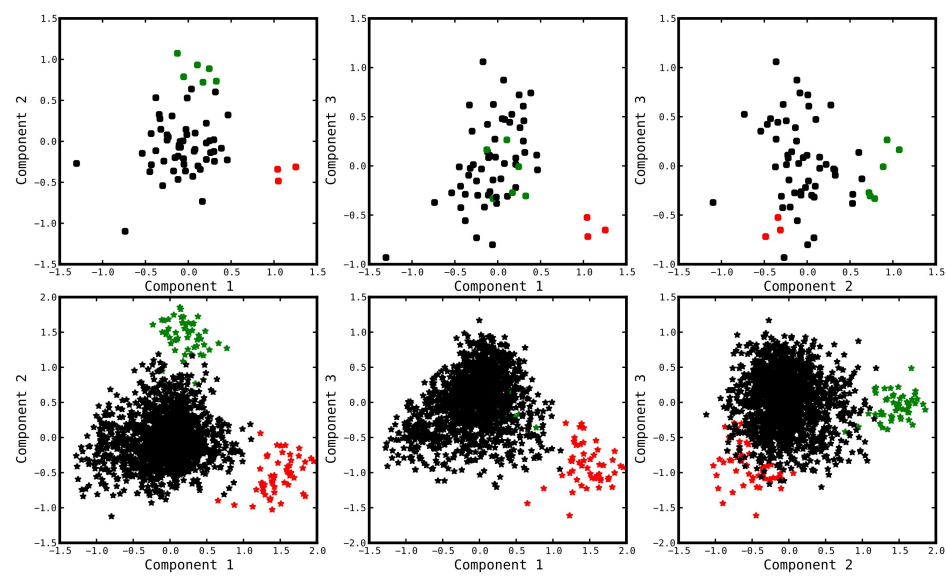

B
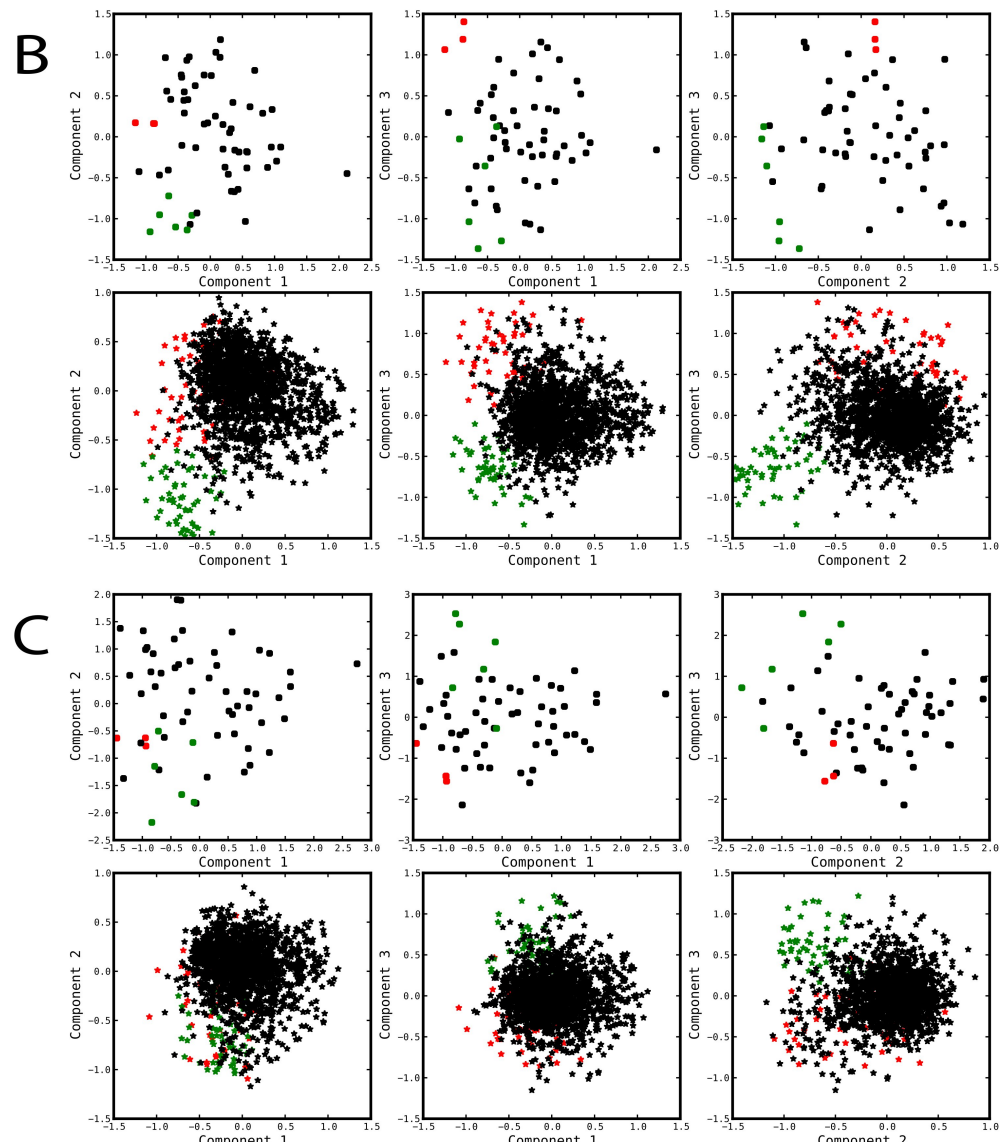

FIG S3. Low-dimensional representation of samples and variables from the human cell cultures microarray data set, obtained by CUMBIA using $K=3$ (panel $A$ ), $K=10$ (panel B) and $K=30$ (panel $C$ ). Green markers represent samples from umbilical artery endothelial cells, and the 50 variables with the highest discriminative power for these samples compared to the rest. Similarly, red markers represent cardiac stromal cell samples and variables. Black markers represent samples from all other subgroups, and the 1,500 variables from the original data set which are least discriminating when contrasting all tissue/cell type combinations in the data set. 
main article. As can be seen in Figure S4, it is considerably more difficult to visually extract the umbilical artery endothelial cell samples and variables (shown in green) when a very small value of $s$ is used.

5. Scree plots for CUMBIA and PCA. Scree plots can be helpful for interpretation of sample configurations obtained by visualization methods such as PCA and CUMBIA. Figures S5, S6 and S7 show scree plots for the three data set analyzed in the main paper. To make them more readable, we have excluded the last, highly negative eigenvalue from CUMBIA and the last eigenvalue (zero) from PCA.

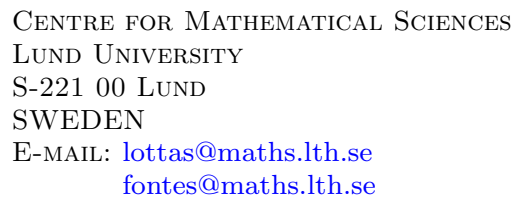



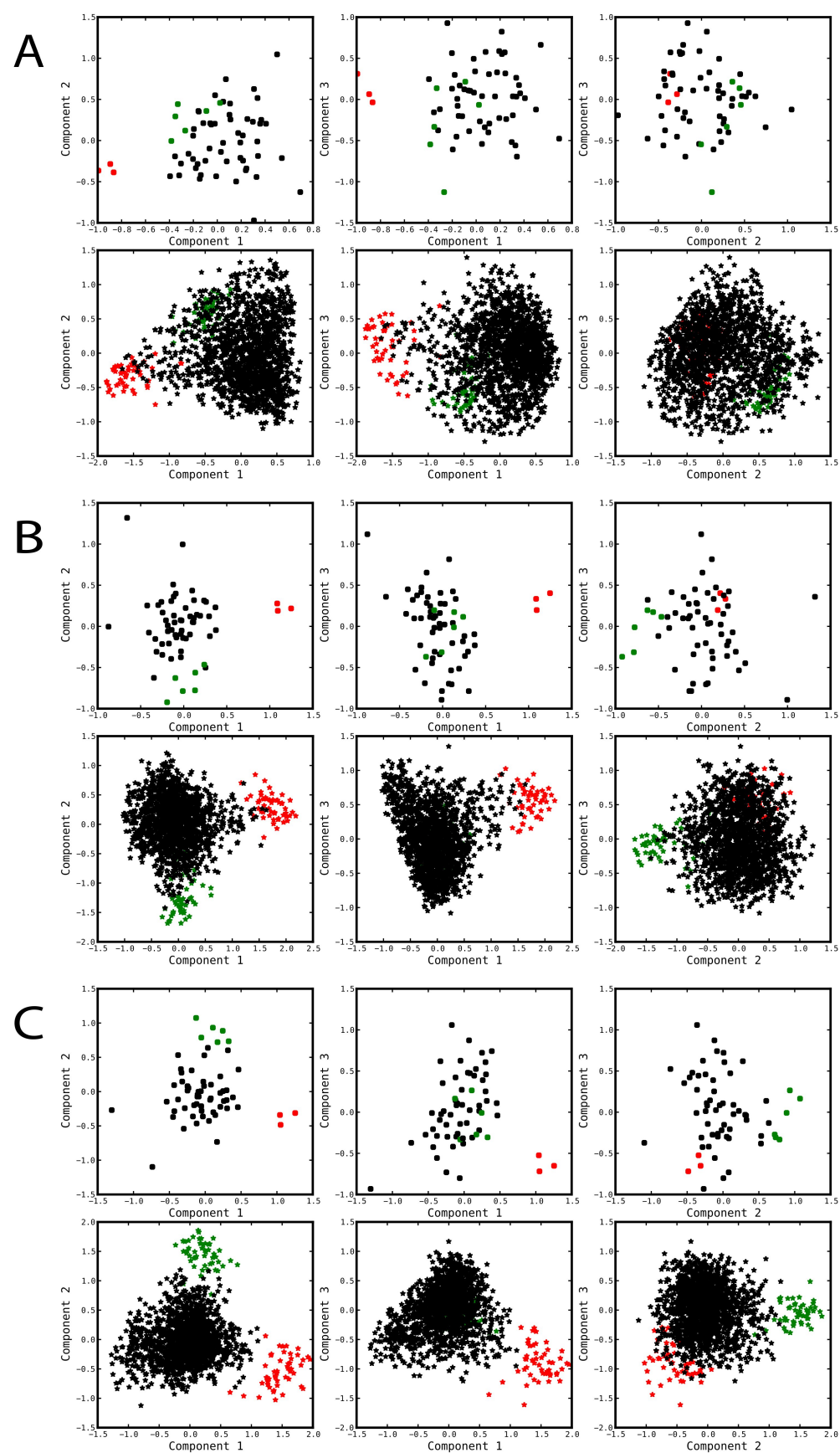

FIG S4. Low-dimensional representation of samples and variables from the human cell cultures microarray data set, obtained by CUMBIA applied to the approximate expression matrix $X_{s}$ for $s=5$ (panel A), $s=15$ (panel B) and $s=r=60$ (panel $C$ ). Green markers represent samples from umbilical artery endothelial cells, and the 50 variables with highest discriminative power for these samples compared to the rest. Similarly, red markers represent cardiac stromal cell samples and variables. Black markers represent samples from all other subgroups, and the 1,500 variables from the original data set which are least discriminating when contrasting all tissue/cell type combinations in the data set. 

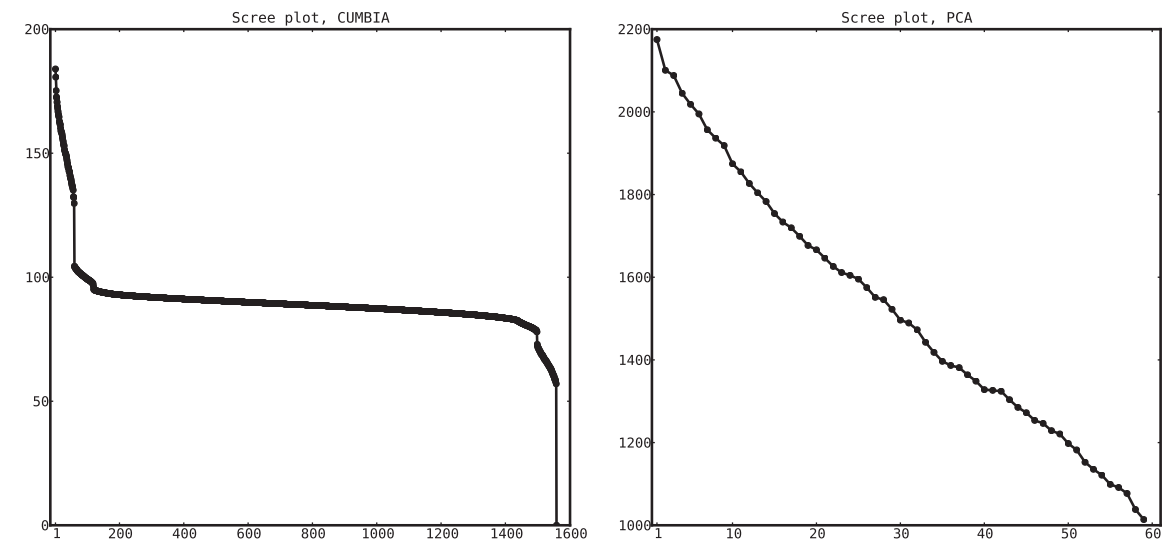

FIG S5. Scree plots obtained by CUMBIA (left panel) and PCA (right panel) for the synthetic data set studied in the main article.
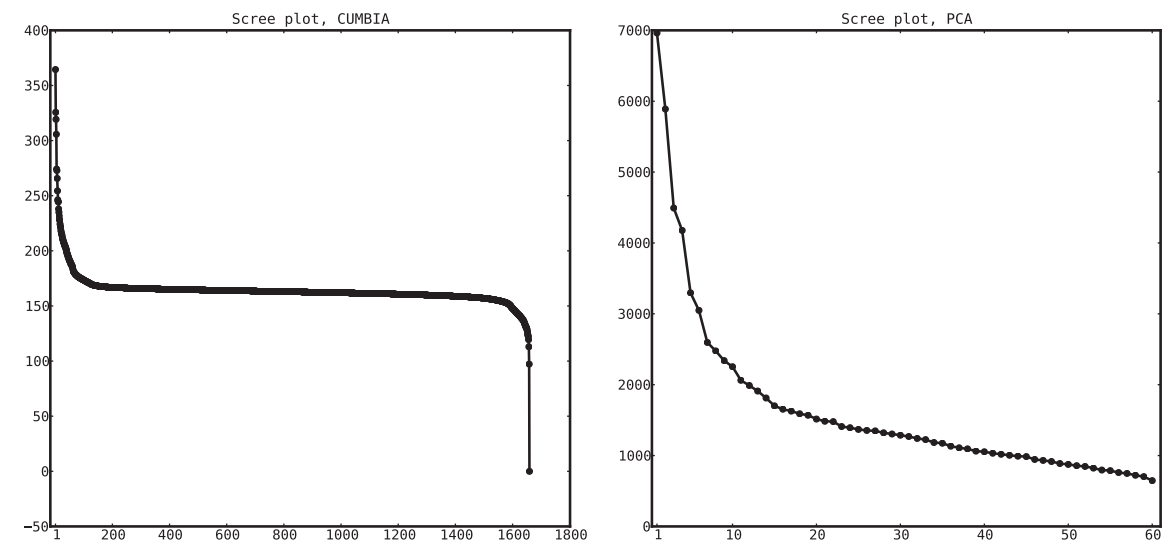

FIG S6. Scree plots obtained by CUMBIA (left panel) and PCA (right panel) for the cell culture data set studied in the main article. 

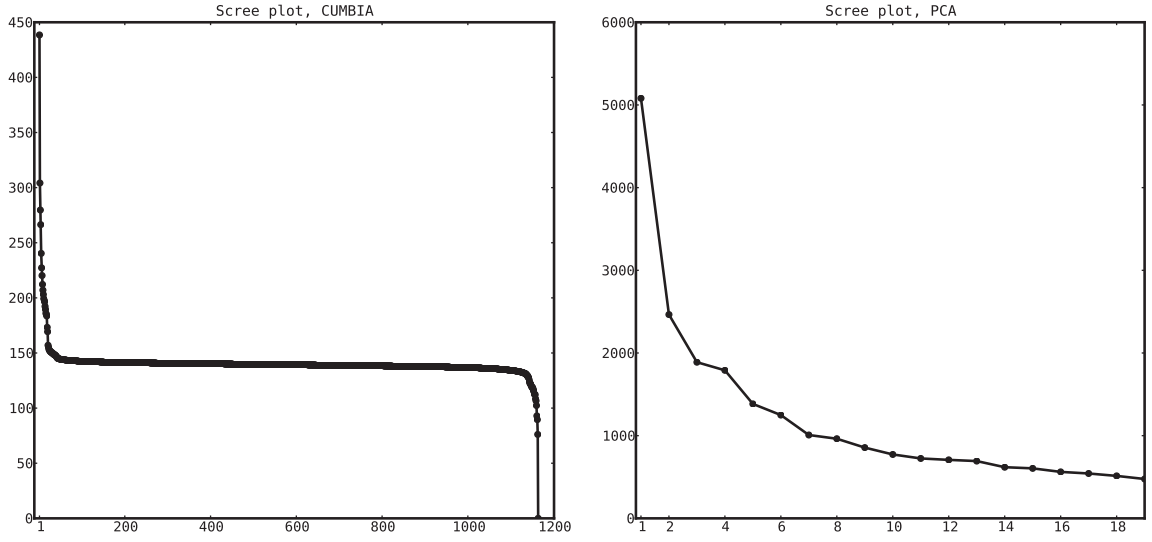

FIG S7. Scree plots obtained by CUMBIA (left panel) and PCA (right panel) for the miRNA data set studied in the main article. 